\title{
Advanced Finite Element Schemes for Multiphysics
}

\author{
Kaltenbacher, Manfred and Triebenbacher, Simon \\ Institute of Mechanics and Mechatronics, Vienna University of Technology \\ Wiedner Hauptstr. 8-10, 1040 Wien, Austria, manfred.kaltenbacher@tuwien.ac.at
}

\begin{abstract}
The design of modern sensors and actuators, which are often Micro - Electro Mechanical - Systems (MEMS), strongly depends on the availability of appropriate computer simulation tools, since the fabrication of each prototype is quite costly. We present an advanved Finite Element (FE) scheme for the design of viscosity sensors, which takes the full coupling between flow dynamics and structural mechanics into account.
\end{abstract}

Keywords: Fluid mechanics, strutural mechanics, Finite Element (FE) method, viscosity sensor

\section{Introduction}

The development of new sensors and actuators is an emerging and highly active research field. Applications are piezoelectric stack actuators, silicon microphones, comb drive acceleration sensors as well as gyroscopes, electrostatic and electromagnetic motors, electromagnetic micro-power generators (energy harvesting), electromechanical as well as optical switches, etc. to name a few $[2,3]$. The sensing and driving mechanism of these smart transducers is mostly based on the interaction between an electric, magnetic or electromagnetic field with a mechanical or a thermal field (electrostatic-mechanical, piezoelectric, magneto-mechanical, electricthermal-mechanical coupling, etc.).

In most cases, the fabrication of prototypes within the design process of sensors and actuators is a lengthy and costly task. Therefore, the need for computer modeling tools capable of precisely simulating the multi-field interactions is increasing. In order to achieve reliable virtual prototyping of sensors and actuators the following main challenges have to be addressed: (1) Solving coupled field problems (multi-physics); (2) Precise constitutive laws for smart materials including the material parameter determination; (3) Efficient numerical schemes for solving the coupled sys- tem of partial differential equations (PDEs); (4) Shape and topology optimization. The accurate modeling of sensors and actuators is very challenging in virtue of the mechanicalelectrical (magnetic) coupling effects and the nonlinearities (e.g. geometric nonlinearity, electrostatic / magnetic force). Effects such as the non-ideal boundary conditions, fringing fields, pre-deformation due to the initial stresses, non-homogeneous structures and moving / deforming bodies up to contact further complicate the modeling [2]. Therefore, only advanced numerical FE schemes are capable to achieve realistic simulation, e.g., [4]. In this sense, the numerical simulation of viscosity sensors is such an example, where standard FE schemes will not work. Therefore, this contribution concentrates on the mathematical modeling, the FE formulation and the numerical simulation of such devices. Thereby, we model the fluid by linearized Navier-Stokes equations and solve the resulting partial differential equations by TaylorHood finite elements. The vibration of the cantilever is computed by Lagrange finite elements.

\section{Governing Equations}

There has been a growing interest in on-line detection of liquid viscosity since it is a crit- 
ical parameter sensitive to material property changes caused by chemical reaction, solidification, gelation, and/or deposition of substances. Thereby, dynamically driven microcantilevers are excited into resonance in the out-of-plane flexural mode. The excitation mechanism reaches from piezoelectric over electro-thermal to electromagnetic principles (see, e.g., $[6,8]$. Here, we just focus on the precise modeling of the interaction of the mechanical vibration (excited by any actuator principle) with the ambient fluid. Therefore, we have to model the mechanical and the flow field as well as their interaction.

\section{Flow Dynamics}

The linearized form of the Navier-Stokes equations can be used to model the fluid. Thereby, the fluid can be considered incompressible as the wavelength of the microcantilever's vibration strongly exceeds the width of the microcantilever, which is the dominant length scale in the flow [1]. The velocity gradient of the fluid is related to the velocity gradient of the vibrating microcantilever. Since it is assumed that the microcantilever's amplitude of vibration is far smaller than any length scale in the microcantilever's geometry, the velocity gradient of the beam and fluid can be considered small, and we can neglect the convective term in the Navier-Stokes equations. Thus, the equation of motion for the fluid is given by

$$
\begin{aligned}
\rho_{\mathrm{f}} \frac{\partial \boldsymbol{v}}{\partial t}+\nabla p-\mu \Delta \boldsymbol{v} & =\mathbf{0}, \\
\nabla \cdot \boldsymbol{v} & =0 .
\end{aligned}
$$

In Eqs. (1) and (2) $\boldsymbol{v}$ denotes the fluid velocity, $p$ the pressure, $\rho_{\mathrm{f}}$ the mean density of the fluid and $\mu$ the dynamic viscosity of the fluid.

The first term on the left hand side of (1) is the term related to the fluid's inertial forces, and the third term on the left hand side is the term related to the fluid's viscous forces. The Reynolds number, in this form sometimes called the nondimensional frequency, can then be found by taking the ratio of these terms resulting for the cantilever in [7]

$$
R e=\frac{\rho_{\mathrm{f}} \omega b^{2}}{4 \mu} .
$$

In (3) $\omega$ denotes the angular frequency and $b$ the width of the cantilever. Furthermore, the properties of the medium of operation, along with the excitation angular frequency $\omega$, will determine the skin depth of the liquid layer surrounding the vibrating beam. The skin depth or boundary layer thickness, denoted by $\delta$, is defined as the distance over which the fluid velocity decays to $1 / e$ of its maximum value. The larger the skin depth, the larger the amount of fluid excited by the vibrating beam. Physically, the boundary layer thickness can be thought of as the amount of fluid trapped in the vortex created by the vibrating microcantilever. The boundary layer thickness can be found as [5]

$$
\delta=\sqrt{\frac{2 \mu}{\rho_{\mathrm{f}} \omega}} .
$$

\section{Structural Mechanics}

To model the structural vibration of the cantilever a linear elasticity model is applied given by Navier's equation

$$
\rho_{\mathrm{s}} \frac{\partial^{2} \boldsymbol{u}}{\partial t^{2}}-\nabla \cdot \boldsymbol{\sigma}_{\mathrm{s}}=\boldsymbol{f}_{\mathrm{V}}
$$

with the Cauchy stress tensor $\boldsymbol{\sigma}_{\mathrm{s}}$, the displacement $\boldsymbol{u}$, any volume force $\boldsymbol{f}_{\mathrm{V}}$, and the solid density $\rho_{\mathrm{s}}$. By introducing the tensor of elasticity $\boldsymbol{c}$ and the tensor of linear strain $\boldsymbol{S}$, Hook's law may be expressed by

$$
\sigma_{\mathrm{s}}=c S .
$$

Furthermore, the linear strain-displacement reads as

$$
\boldsymbol{S}=\frac{1}{2}\left(\nabla \boldsymbol{u}+(\nabla \boldsymbol{u})^{t}\right)=\mathcal{B} \boldsymbol{u}
$$

with the differential operator $\mathcal{B}$ computed by

$$
\mathcal{B}=\left(\begin{array}{cccccc}
\frac{\partial}{\partial x} & 0 & 0 & 0 & \frac{\partial}{\partial z} & \frac{\partial}{\partial y} \\
0 & \frac{\partial}{\partial y} & 0 & \frac{\partial}{\partial z} & 0 & \frac{\partial}{\partial x} \\
0 & 0 & \frac{\partial}{\partial z} & \frac{\partial}{\partial y} & \frac{\partial}{\partial x} & 0
\end{array}\right)^{t}
$$


Inserting equation (6) and (7) into (5) results in the final partial differential equation (PDE) for linear elasticity

$$
\rho_{\mathrm{s}} \frac{\partial^{2} \boldsymbol{u}}{\partial t^{2}}-\mathcal{B}^{t} \boldsymbol{c} \mathcal{B} \boldsymbol{u}=\boldsymbol{f}_{\mathrm{V}} .
$$

\section{Coupling}

For a correct representation of the fluidstructure interaction two conditions must be fulfilled at the common interface $\Gamma_{\mathrm{fs}}$ between fluid and solid. First, the fluid velocity and structural velocity have to be identical given by

$$
\boldsymbol{v}=\frac{\partial \boldsymbol{u}}{\partial t} \quad \text { on } \Gamma_{\mathrm{fs}} .
$$

This implies that the fluid adheres to the structure. For a fixed wall this corresponds to a so called no slip conditions. The second condition is the continuity of stress in normal direction along the interface, meaning fluid stress $\boldsymbol{\sigma}_{\mathrm{f}}$ and solid stress $\boldsymbol{\sigma}_{\mathrm{s}}$ have to coincide which is enforced by

$$
\boldsymbol{\sigma}_{\mathrm{s}} \cdot \boldsymbol{n}=\boldsymbol{\sigma}_{\mathrm{f}} \cdot \boldsymbol{n} \quad \text { on } \Gamma_{\mathrm{fs}} .
$$

The acting fluid forces can be split up into a pressure and a surface viscous stress component

$$
\begin{array}{r}
\boldsymbol{f}_{\mathrm{fs}}=\int_{\Gamma_{\mathrm{fs}}} \boldsymbol{\sigma}_{\mathrm{f}} \cdot \boldsymbol{n} \mathrm{d} s=\underbrace{\int_{\Gamma_{\Gamma_{\mathrm{fs}}}}-p \boldsymbol{I} \cdot \boldsymbol{n} \mathrm{d} s}_{\text {pressure force }} \\
+\underbrace{\int_{\Gamma_{\mathrm{fs}}} \mu\left(\nabla \boldsymbol{v}+(\nabla \boldsymbol{v})^{t}\right) \cdot \boldsymbol{n} \mathrm{d} s}_{\text {viscous force }} .
\end{array}
$$

\section{FE Formulation}

The first step within each FE formulation is to derive the weak formulation. Therewith, we multiply (1), (2) with appropriate test function $(\boldsymbol{\psi}, \varphi)$ and integrate over the whole com- putational domain $\Omega_{\mathrm{f}}$ for the fluid

$$
\begin{array}{r}
\int_{\Omega_{\mathrm{f}}} \rho_{\mathrm{f}} \boldsymbol{\psi} \cdot \frac{\partial \boldsymbol{v}}{\partial t} \mathrm{~d} x-\int_{\Omega_{\mathrm{f}}} \boldsymbol{\psi} \cdot \mathcal{B}^{t} \boldsymbol{\sigma}_{\mathrm{f}} \mathrm{d} x=\mathbf{0}, \\
\int_{\Omega_{\mathrm{f}}} \varphi \nabla \cdot \boldsymbol{v} \mathrm{d} x=0 .
\end{array}
$$

In (12) we have used the fluid stress $\boldsymbol{\sigma}_{\mathrm{f}}$ (see (11)). In a next step, we perform an integration by parts for the second term in (12) resulting in

$$
\begin{gathered}
\int_{\Omega_{\mathrm{f}}} \boldsymbol{\psi} \cdot \mathcal{B}^{t} \boldsymbol{\sigma}_{\mathrm{f}} \mathrm{d} x=-\int_{\Omega_{\mathrm{f}}}(\mathcal{B} \boldsymbol{\psi})^{t} \cdot \boldsymbol{\sigma}_{\mathrm{f}} \mathrm{d} x \\
+\int_{\Gamma_{\mathrm{fs}}} \boldsymbol{\psi} \cdot \boldsymbol{\sigma}^{t} \boldsymbol{n}_{\mathrm{fs}} \mathrm{d} s+\int_{\Gamma_{\mathrm{f}}} \boldsymbol{\psi} \cdot \boldsymbol{\sigma}^{t} \boldsymbol{n}_{\mathrm{f}} \mathrm{d} s
\end{gathered}
$$

with $\Gamma_{\mathrm{fs}}$ the interface between the fluid and the solid, $\Gamma_{\mathrm{f}}$ the outer boundary limiting the computational domain $\Omega_{\mathrm{f}}$, and $\boldsymbol{n}_{\mathrm{fs}}$ the normal vector at the coupling interface pointing from the flow region into the solid region. Substituting (14) into (12) results in the weak formulation for the linearized Navier- Stokes equations

$$
\begin{array}{r}
\int_{\Omega_{\mathrm{f}}} \rho_{\mathrm{f}} \boldsymbol{\psi} \cdot \frac{\partial \boldsymbol{v}}{\partial t} \mathrm{~d} x+\int_{\Omega_{\mathrm{f}}}(\mathcal{B} \boldsymbol{\psi})^{t} \cdot \boldsymbol{\sigma}_{\mathrm{f}} \mathrm{d} x \\
-\int_{\Gamma_{\mathrm{fs}}} \boldsymbol{\psi} \cdot \boldsymbol{\sigma}^{t} \boldsymbol{n}_{\mathrm{fs}} \mathrm{d} s-\int_{\Gamma_{\mathrm{f}}} \boldsymbol{\psi} \cdot \boldsymbol{\sigma}^{t} \boldsymbol{n}_{\mathrm{f}} \mathrm{d} s=\mathbf{0} \\
\int_{\Omega_{\mathrm{f}}} \varphi \nabla \cdot \boldsymbol{v} \mathrm{d} x=0 .(16)
\end{array}
$$

Denoting with $\phi$ the test function for the mechanical PDE, and performing similar steps, we arrive at the weak formulation for mechanics

$$
\begin{array}{r}
\int_{\Omega_{\mathrm{s}}} \rho_{\mathrm{s}} \boldsymbol{\phi} \cdot \frac{\partial^{2} \boldsymbol{u}}{\partial t^{2}} \mathrm{~d} x+\int_{\Omega_{\mathrm{s}}}\left(\mathcal{B}^{t} \boldsymbol{\phi}\right)^{t} \boldsymbol{c} \mathcal{B} \boldsymbol{u} \mathrm{d} x \\
+\int_{\Gamma_{\mathrm{fs}}} \boldsymbol{\phi} \cdot \boldsymbol{\sigma}_{\mathrm{s}}^{t} \boldsymbol{n}_{\mathrm{fs}} \mathrm{d} s-\int_{\Gamma_{\mathrm{s}}} \boldsymbol{\phi} \cdot \boldsymbol{\sigma}_{\mathrm{s}}^{t} \boldsymbol{n}_{\mathrm{s}} \mathrm{d} s \\
=\int_{\Omega_{\mathrm{s}}} \boldsymbol{\phi} \cdot \boldsymbol{f}_{\mathrm{V}} \mathrm{d} x
\end{array}
$$


Now, we introduce a Lagrange multiplier $\boldsymbol{\lambda}$ to guarantee the coupling condition according to (10)

$$
\boldsymbol{\lambda}=-\boldsymbol{\sigma}_{\mathrm{s}} \cdot \boldsymbol{n}_{\mathrm{fs}}=-\boldsymbol{\sigma}_{\mathrm{f}} \cdot \boldsymbol{n}_{\mathrm{fs}} .
$$

Furthermore, the coupling condition (9) will be fulfilled in our formulation in a weak sense, thus by

$$
\int_{\Gamma_{\mathrm{fs}}}\left(\boldsymbol{v}-\frac{\partial \boldsymbol{u}}{\partial t}\right) \cdot \boldsymbol{\mu} \mathrm{d} s=0 .
$$

Finally, we can combine the above equations to achieve at the overall formulation

$$
\begin{aligned}
& \int_{\Omega_{\mathrm{f}}} \rho_{\mathrm{f}} \boldsymbol{\psi} \cdot \frac{\partial \boldsymbol{v}}{\partial t} \mathrm{~d} x \\
& +\int_{\Omega_{\mathrm{f}}}(\mathcal{B} \boldsymbol{\psi})^{t} \cdot\left(-p \boldsymbol{I}+\mu\left(\nabla \boldsymbol{v}+(\nabla \boldsymbol{v})^{t}\right)\right) \mathrm{d} x \\
& -\int_{\Gamma_{\mathrm{fs}}} \boldsymbol{\psi} \cdot \boldsymbol{\lambda} \mathrm{d} s=\int_{\Gamma_{\mathrm{f}}} \boldsymbol{\psi} \cdot \boldsymbol{\sigma}^{t} \boldsymbol{n}_{\mathrm{f}} \mathrm{d} s \\
& \int_{\Omega_{\mathrm{f}}} \varphi \nabla \cdot \boldsymbol{v} \mathrm{d} x=0 \\
& \int_{\Omega_{\mathrm{s}}} \rho_{\mathrm{s}} \phi \cdot \frac{\partial^{2} \boldsymbol{u}}{\partial t^{2}} \mathrm{~d} x \\
& +\int_{\Omega_{\mathrm{s}}}\left(\mathcal{B}^{t} \phi\right)^{t} \boldsymbol{c} \mathcal{B} \boldsymbol{u} \mathrm{d} x+\int_{\Gamma_{\mathrm{fs}}} \phi \cdot \boldsymbol{\lambda} \mathrm{d} s \\
& -\int_{\Gamma_{\mathrm{s}}} \boldsymbol{\phi} \cdot \boldsymbol{\sigma}_{\mathrm{s}}^{t} \boldsymbol{n}_{\mathrm{s}} \mathrm{d} s=\int_{\Omega_{\mathrm{s}}} \boldsymbol{\phi} \cdot \boldsymbol{f}_{\mathrm{V}} \mathrm{d} x \\
& \int_{\Gamma_{\mathrm{fs}}}\left(\boldsymbol{v}-\frac{\partial \boldsymbol{u}}{\partial t}\right) \cdot \mu \mathrm{d} s=0
\end{aligned}
$$

The space discretization is performed with Lagrange FE basis functions. Thereby, we have to use Taylor-Hood elements for the discretization of the flow velocity and pressure. This means that we use second order basis functions for the flow velocity $\mathbf{v}$ but first order basis functions for the pressure. Furthermore, we choose second order basis functions for the mechanical displacement $\boldsymbol{u}$, and first order basis functions for the Lagrange multiplier $\boldsymbol{\lambda}$. In a last step, we do a Fourier transform of the semi-discrete Galerkin formulation, which results in a complex algebraic system of equations.

\section{Numerical Results}

As a numerical example to demonstrate the applicability of the developed coupled FE scheme, we investigate a MEMS cantilever. The setup is displayed in Fig. 1, which consists of the cantilever and the ambient fluid.

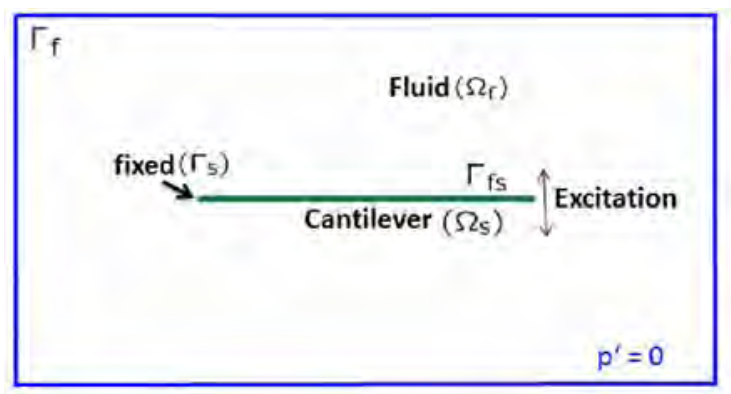

Figure 1: Computational setup.

To correctly resolve the boundary layer, we use a quite fine mesh around the cantilever which we then coarse towards the boundary $\Gamma_{\mathrm{f}}$ as shown in Fig. 2.

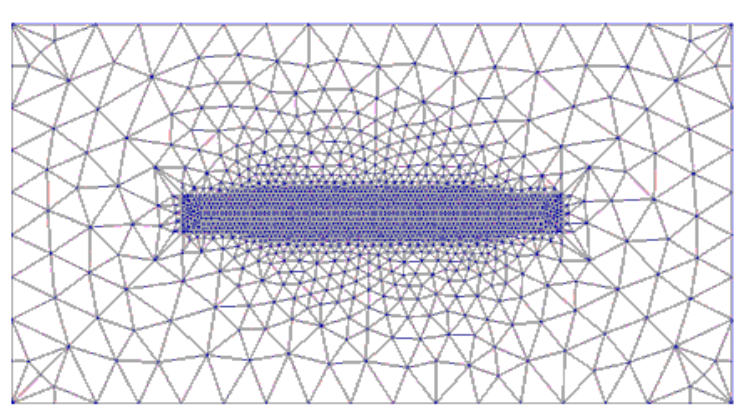

Figure 2: Computational mesh

In a first step, we use the properties of air and perform a coupled simulation at the first eigen-frequency of the cantilever, which is at about $18 \mathrm{kHz}$. Figure 3 displays the imaginary part of the flow velocity around the tip of the cantilever, and Fig. 4 the generated pressure distribution in the whole computational domain of the fluid. 


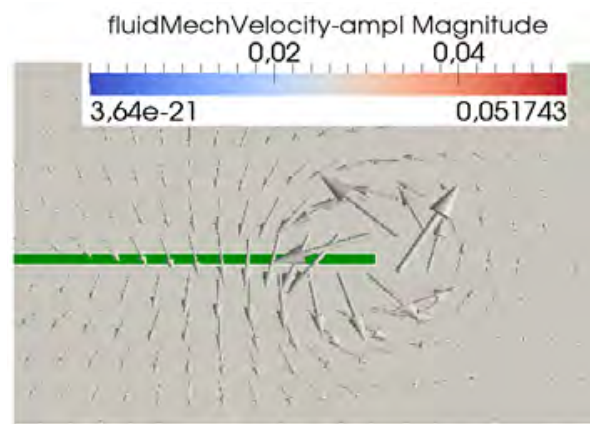

Figure 3: Imaginary part of flow velocity near the tip of the cantilever (zoomed).

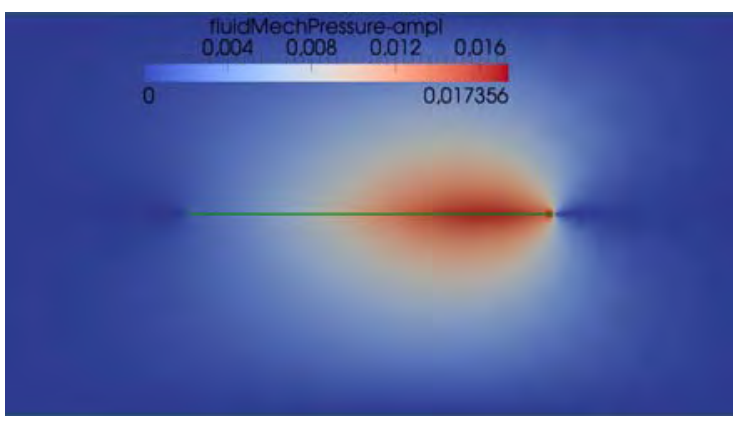

Figure 4: Resulting flow pressure, when the cantilever operates at the first eigen-frequency

Finally, we perform a harmonic analysis for a frequency range around the first eigenfrequency and compare the tip displacement once obtained just by a mechanical computation (no ambient fluid being taken into account) and once for the fully coupled one, which takes the ambient air into account. It should be noted that we use a Rayleigh damping model with a loss factor of 0.05 for the cantilever.

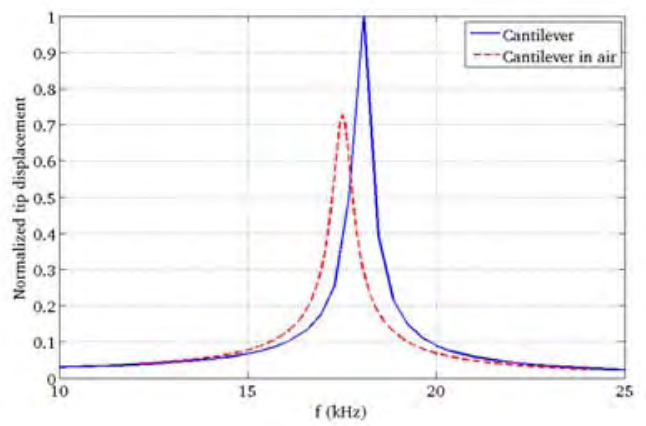

Figure 5: Mechanical displacement of the cantilever around the first eigen-frequency.
Figure 5 clearly demonstrates the influence of the ambient air on the amplitude and shift in the eigen-frequency.

\section{References}

[1] S. Basak and A. Raman. Hydrodynamic Loading of Microcantilevers Vibrating in Viscous Fluids. J. Appl. Phys., 2006.

[2] W. C. Chuang, H. L. Lee, P. Z. Chang, and Y. C. Hu. Review on the Modeling of Electrostatic MEMS Sensors. Sensors, 10(6)::6149-6171, 2010.

[3] M. Kaltenbacher. Numerical Simulation of Mechatronic Sensors and Actuators. Springer, Berlin, 2. edition, 2007. ISBN: 978-3-540-71359-3.

[4] S. Prasad, A. Sumant, A. C. Cangellaris, and N. R. Aluru. A conformal mappingbased approach for fast two-dimensional FEM electrostatic analysis of MEMS devices. Int. J. Numer. Model., 24:194-206, 2011.

[5] L. Rosenhead. Laminar Boundary Layers. Oxford: Clarendon press, 1963.

[6] W. Y. Shih, X. Li, H. Gu, W. H. Shih, and I. A. Aksay. Simultaneous liquid viscosity and density determination with piezoelectric unimorph cantilevers. J. Appl. Phys., 89:1497-1505, 2001.

[7] E. Tuck. Calculation of Unsteady Flows due to Small Motions of Cylinders in a Viscous Fluid. Journal of Engineering Mathematics, 3(1):29-44, 1969.

[8] B. Weiss, E. K. Reichel, and B. Jakoby. Modeling of a clamped-clamped beam vibrating in a fluid for viscosity and density sensing regarding compressibility. Sensors and Actuators A, 143:293-301, 2008. 